Musées, Patrimoine et Culture scientifiques et techniques

$160 \mid 2015$

juillet-août 2015

\title{
Le projet de cité de l'économie et de la monnaie à Paris
}

\section{Charlotte Drahé}

\section{OpenEdition \\ Journals}

Édition électronique

URL : http://journals.openedition.org/ocim/1548

DOI : $10.4000 /$ ocim. 1548

ISSN : 2108-646X

\section{Éditeur}

OCIM

\section{Édition imprimée}

Date de publication : 1 juillet 2015

Pagination : 22-27

ISSN : 0994-1908

\section{Référence électronique}

Charlotte Drahé, «Le projet de cité de l'économie et de la monnaie à Paris », La Lettre de l'OCIM [En ligne], 160 | 2015, mis en ligne le 01 juillet 2016, consulté le 19 avril 2019. URL : http:// journals.openedition.org/ocim/1548; DOI : 10.4000/ocim.1548

Ce document a été généré automatiquement le 19 avril 2019.

Tous droits réservés 


\title{
Le projet de cité de l'économie et de la monnaie à Paris
}

\author{
Charlotte Drahé
}

La façade du bâtiment qui devrait accueillir la Cité de l'économie et de la monnaie.

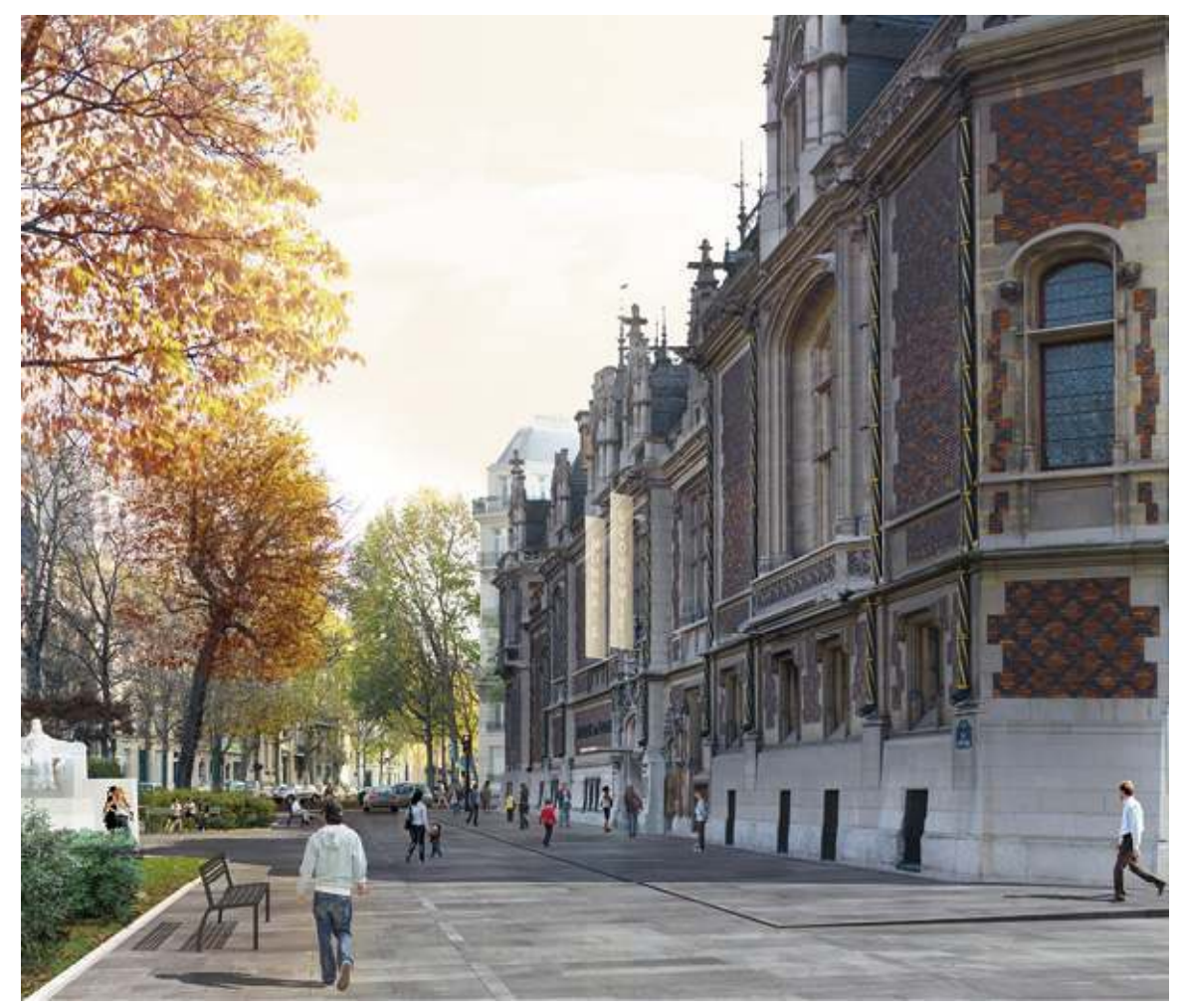

(c) Atelier Lion

1 Le projet de la Cité de l'économie et de la monnaie qui devrait ouvrir ses portes dans quelques années à Paris sera dédié à la culture économique et devrait s'installer dans le 
17e arrondissement, au sein de l'hôtel Gaillard, Monument historique du XIXe siècle. Cette citée vise à sensibiliser un large public, particulièrement les jeunes, aux enjeux économiques contemporains et aux outils qui permettent de les comprendre. Elle propose d'assurer un accompagnement des citoyens dans la compréhension du monde économique qui les entoure.

\section{Contexte de la naissance du projet}

2 Posséder quelques bases en culture économique est essentiel pour mieux comprendre l'actualité et les débats économiques, et mieux en maîtriser les enjeux pour ses choix personnels. Plus largement, une meilleure culture économique de tous contribue à améliorer le fonctionnement de l'économie et l'efficacité des politiques mises en œuvre. Depuis quelques années, des enquêtes auprès des Français creusent ces questions. Elles illustrent une attention nouvelle, notamment des pouvoirs publics, qui tentent de mieux cerner le niveau de connaissance des citoyens en économie, cette mobilisation pouvant s'expliquer par les nombreuses questions suscitées par la crise que nous traversons. Citons à cet égard les enquêtes réalisées par la Banque de France sur les connaissances économiques des Français ou les travaux de la commission Guesnerie au ministère de l'Éducation nationale sur la révision des programmes scolaires en économie ${ }^{1}$.

3 L'intérêt avéré pour ce sujet de société doit être confronté aux résultats des enquêtes réalisées. En effet, elles indiquent que les Français connaissent mal l'économie et qu'ils éprouvent face à cette dernière un sentiment de rejet car ils jugent l'information économique complexe, floue, éloignée de leur quotidien. Pourtant, les séries ES ou économie-gestion (STMG) rencontrent un certain succès au lycée et $20 \%$ des lycéens se dirigent en série ES et $12 \%$ en STMG (voir Repères et références statistiques sur les enseignements, la formation et la recherche, 2013, p. 123). Par ailleurs de nombreuses publications grand public traitant de sujets économiques sont éditées chaque année. Néanmoins, les personnes interrogées ont conscience de l'impérieuse nécessité de mieux comprendre cette information, car l'économie transforme et impacte leur vie quotidienne (budget des ménages, emploi, immobilier) via notamment les décisions de politique économique, nationales et internationales. Ainsi, au-delà d'une première réticence, ces résultats traduisent une véritable volonté de la population d'en savoir plus à l'égard de l'économie. Mieux la connaître, en maîtriser les concepts clefs et faire en sorte que leurs enfants soient mieux armés qu'eux, sont les principaux souhaits qu'expriment les enquêtés.

4 Face à cette situation, des initiatives ont vu le jour, notamment : la création en 2006, sous l'égide de l'Autorité des Marchés Financiers, de l'Institut pour l'éducation financière du grand public, partenaire du projet de la Cité de l'économie et de la monnaie, la conduite de projets pédagogiques dans le domaine bancaire avec des scolaires ("La Banque, ma banque et moi", "Les clefs de la Banque" avec la Fédération Bancaire Française) et surtout, depuis 1966, la possibilité de choisir une option "sciences économiques et sociales" dès l'entrée en seconde et de poursuivre ensuite dans une série dédiée.

5 Parallèlement, l'offre d'événements présentant un programme de conférences avec des sujets économiques s'adressant à un plus large public, s'est renforcée ces dernières années. On peut citer les Journées de l'Économie à Lyon (depuis 2008 au mois d'octobre/ novembre), le Printemps de l'Économie à Paris (depuis 2013 au mois d'avril), les 
Rencontres des SES à Paris (depuis 2012 au mois de janvier), le Festival de l'Économie à Annecy (depuis 2010 au mois de novembre), le Festival de Géopolitique à Grenoble (depuis 2009 au mois de mars/avril), le Festival international de Géographie à Saint-Dié-desVosges (depuis 1990 au mois d'octobre) et les Rendez-vous de l'Histoire à Blois (depuis 1998 au mois d'octobre) qui pour la première fois en 2014 ont proposé une "section économie" entièrement dédiée à ces problématiques. La Cité de l'économie et de la monnaie a d'ailleurs participé à plusieurs de ces événements en organisant des conférences-débat ${ }^{2}$.

6 C'est dans ce contexte que la création d'un lieu entièrement dédié à l'économie, ouvert à tous les publics, accessible, éducatif et citoyen, est apparue utile et pertinente. Piloté par la Banque de France, le projet de création d'une Cité de l'économie et de la monnaie a été lancé en 2011 avec un concours international remporté par Ateliers Lion pour l'architecture et l'agence Confino pour la scénographie. Les compétences nécessaires à la conduite de ce chantier muséal ont été réunies au sein d'une équipe de direction de projet. Intégrée à la Direction des Études et des Relations Internationales de la Banque de France, la direction du projet s'organise autour de deux services, celui des contenus pédagogiques et muséographiques et celui des moyens. Le premier regroupe les contenus pédagogiques, la conception muséographique, et le développement des publics, le second la gestion du chantier architectural, le site Internet et les fonctions supports administratives.

7 Comme la plupart des pays européens, la France possède des espaces muséaux dédiés à des collections numismatiques. Il convient de souligner l'existence, à Paris, du médaillier de la Bibliothèque Nationale de France - site Richelieu - et celui de la Monnaie de Paris, institutions partenaires du projet de la Cité de l'économie et de la monnaie. Chez nos voisins européens, des espaces muséaux similaires, parfois élargis à la politique monétaire et plus rarement à l'économie en général, ont été créés par les banques centrales (musée de la Banque centrale d'Allemagne, Geldmuseum; musée de la Banque centrale d'Angleterre, Bank of England Museum; musée de la Banque Nationale de Belgique; musée de la Banque de Finlande...). Le même constat peut être fait pour le continent américain où les musées monétaires sont bien représentés aux États-Unis. Une mention particulière doit être attribuée au musée d'économie créé en 2006 par la Banque centrale du Mexique ${ }^{3}$, le Museo Interactivo de Economia, première institution culturelle dédiée à l'économie en général où la pédagogie et l'interactivité sont véritablement au cœur du propos. 
Projet pour le secteur consacré aux échanges commerciaux.

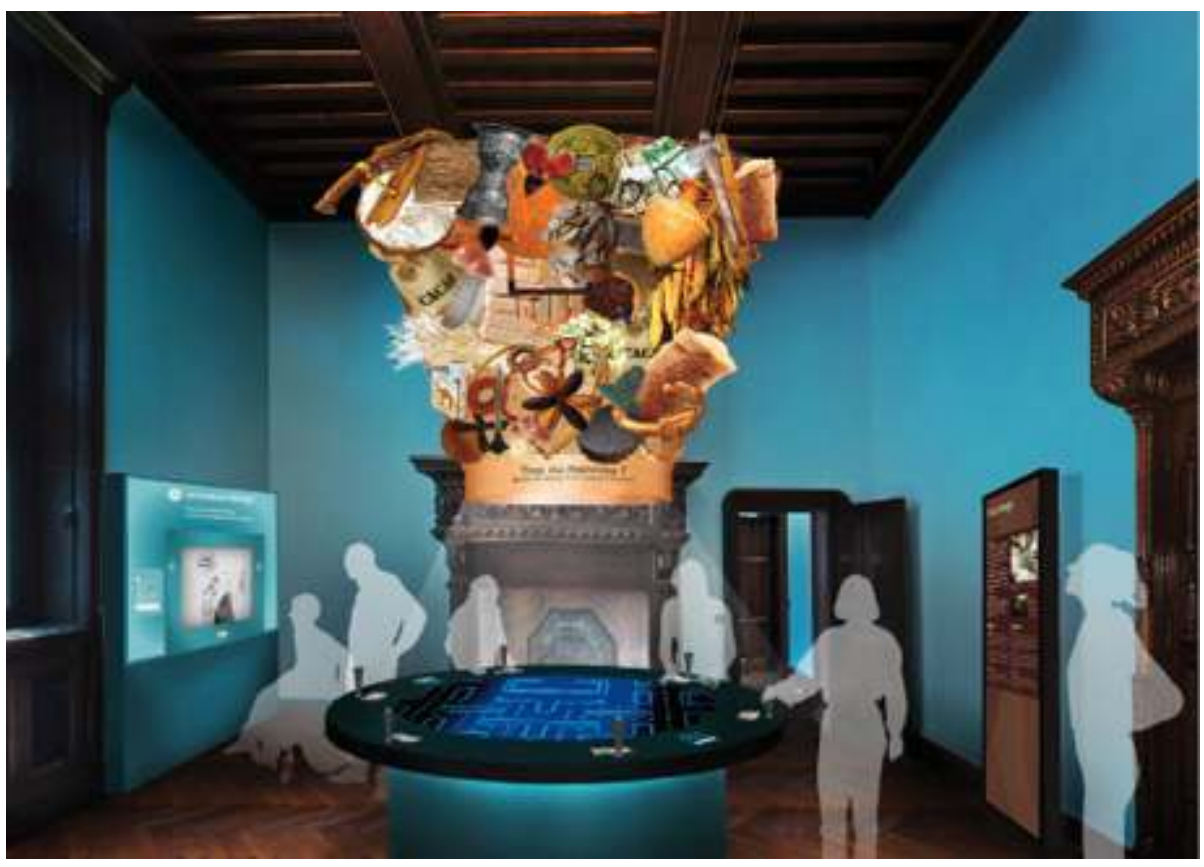

(C) Confino,

\section{La future Cité de l'économie et de la monnaie}

La future Cité de l'économie et de la monnaie s'installera dans l'hôtel Gaillard, Monument historique du XIXe siècle situé sur la plaine Monceau dans le 17e arrondissement de Paris. Remarquable pour son architecture néo-renaissance inspirée des châteaux de Gien et de Blois, cet hôtel particulier présente des décors intérieurs - boiseries, sculptures - de qualité. Une des pièces maîtresses de cet ensemble est la salle des coffres, entourée de douves en eau, accessible par un plancher amovible et dont l'architecture intérieure évoque le style Art déco. Ce bâtiment classé Monument historique a longtemps été l'une des succursales de la Banque de France à Paris. Il sera ouvert pour la première fois au grand public. Entièrement réaménagé par une muséographie contemporaine (qui respectera l'architecture ancienne), il sera également doté de tous les services nécessaires au confort de la visite.

9 Un site Internet (www.citedeleconomie.fr/-Francais) présente le projet et propose de nombreuses rubriques dédiées à la découverte de l'économie : un portail de ressources, des vidéos et jeux pédagogiques, des bibliographies, un focus sur les collections ou encore un espace spécialement dédié aux enseignants.

10 Sur $2400 \mathrm{~m}^{2}$, l'exposition permanente se déploiera à travers six grandes thématiques allant des «Échanges" aux "Acteurs", en passant par les "Marchés» et leurs «Instabilités », jusqu'aux "Régulations ». Ces cinq premières sections inviteront les visiteurs à une découverte progressive des thématiques et mécanismes économiques. Elles offriront également un parcours architectural à travers les deux premiers niveaux du bâtiment, passant par les parties les plus anciennes datant du XIXe siècle et se terminant dans le "Hall Defrasse", ancien espace d'accueil des clients de la succursale, qui fut ajouté au XXe siècle par la Banque de France. Au fil du parcours, des débats filmés, sur 
des sujets d'actualité et confrontant divers points de vue, seront proposés aux visiteurs. Ces espaces formeront également des zones de pause au cœur du parcours.

Une dernière section sera consacrée aux «Trésors ». Installée dans l'ancienne salle des coffres en sous-sol, elle présentera, sur deux niveaux, les collections patrimoniales monétaires appartenant à la Banque de France ainsi que des prêts d'institutions culturelles partenaires (Bibliothèque Nationale de France, Monnaie de Paris, Conservatoire national des Arts et Métiers...). Les collections évoqueront l'évolution des moyens de paiement à travers le monde dans un parcours à la fois chronologique et thématique. La visite de cet espace aura également un intérêt sur le plan architectural ainsi que pour ses aménagements intérieurs, notamment l'entrée dans la salle des coffres qui s'effectue par un plancher amovible actionné par un mécanisme automatique, et son mobilier d'époque.

Deux salles d'expositions temporaires $\left(430 \mathrm{~m}^{2}\right)$ situées au dernier étage du bâtiment pourront traiter de sujets d'actualités ou approfondir certains thèmes présentés dans l'exposition permanente. Elles seront directement accessibles par des ascenseurs situés dans le hall d'accueil du public au rez-de-chaussée.

Par ailleurs, deux salles d'ateliers pédagogiques et un centre de ressources spécialisé en pédagogie de l'économie sont prévus. Ces espaces seront adaptés à des animations encadrées visant à faciliter la découverte et l'appropriation des notions économiques, notamment par les plus jeunes.

Enfin, un hall d'accueil du public, une boutique et un café offriront le confort nécessaire à chacun. La visite pourra se clôturer par la découverte des toits, au dernier niveau de l'hôtel de Gaillard. Les salles d'expositions temporaires et les ateliers pédagogiques offriront une vue et un accès direct à ces espaces aménagés en terrasses.

Projet pour la salle des coffres.

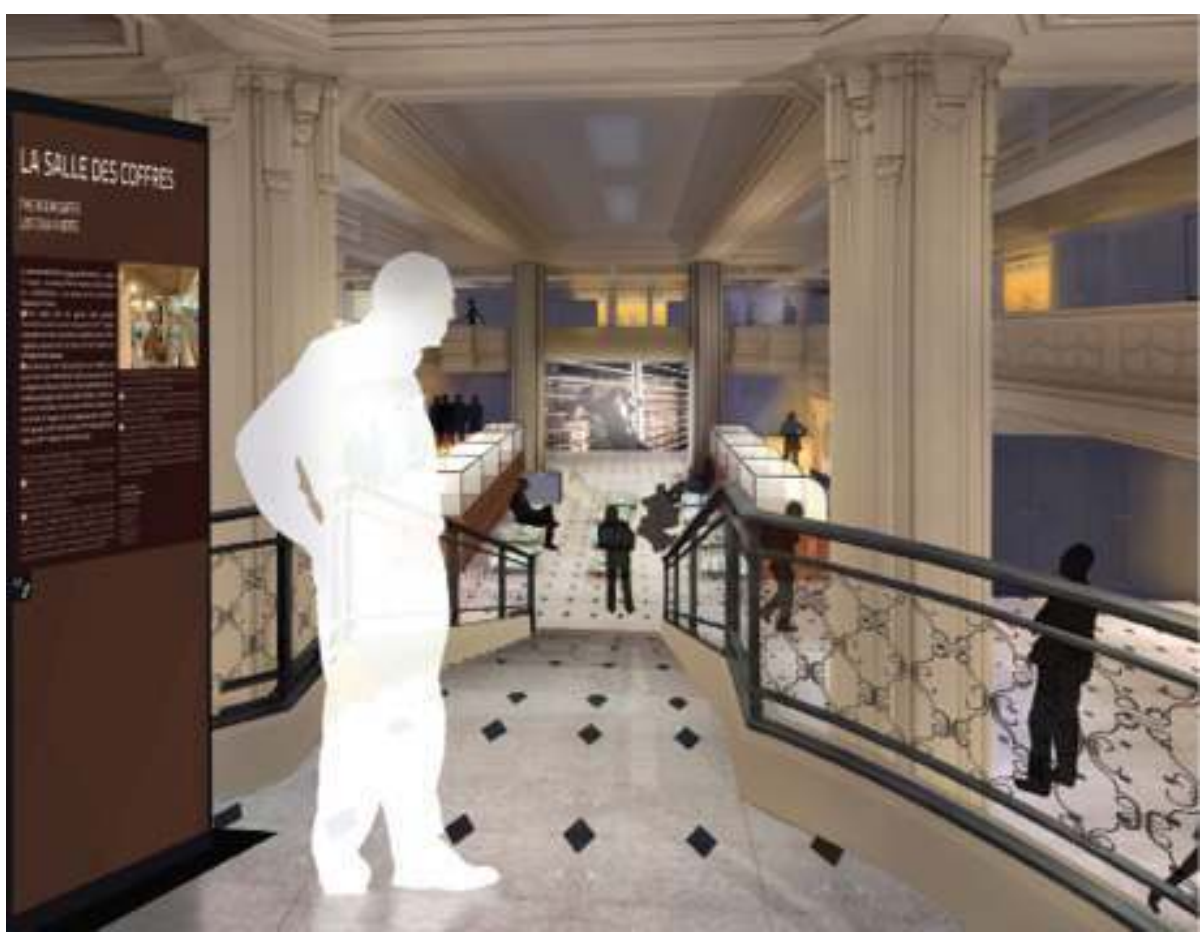

(c) Confino 


\section{Pour qui ?} particulier aux adolescents à partir de 14-15 ans. Les plus jeunes seront également conviés à découvrir la Cité, notamment à travers la visite de son monument et de la salle des coffres où seront exposés les « Trésors ». Les publics individuels, en famille ou entre amis, sont naturellement attendus.

Accueillir une majorité d'adolescents, public complexe à faire venir au musée, est un déf de taille pour cette future institution. Sa médiation cherchera donc à surprendre, à faire participer les publics, notamment à travers des parcours et des ateliers où des techniques proches de celles des jeux de rôles pourraient être employées. Un programme spécifique d'activités sera également attaché au centre de ressources et à l'auditorium formant ainsi une offre culturelle diversifiée développée pour l'ouverture.

L'accessibilité du parcours permanent a été particulièrement travaillée. Il sera traduit en deux langues, l'anglais et l'espagnol. La plupart des espaces muséographiques, grâce à des ascenseurs et rampes d'accès, seront accessibles aux personnes à mobilité réduite. Des outils de médiation spécifiques seront développés pour les personnes sourdes et malentendantes (visioguides en langue des signes française et traduction de tous les multimédia du parcours). Un audioguide en audio description et des stations tactiles réparties dans le parcours permanent seront proposés aux personnes en situation de handicap visuel, ainsi que des médiations humaines adaptées aux personnes en situation de handicap mental.

Enfin, une programmation sera ouverte, avec et pour les chercheurs, économistes et amateurs avertis qui pourront participer, dans l'auditorium, à des conférences sur des thèmes plus pointus.

Cit€co, un "jeu sérieux" pour découvrir l'économie.

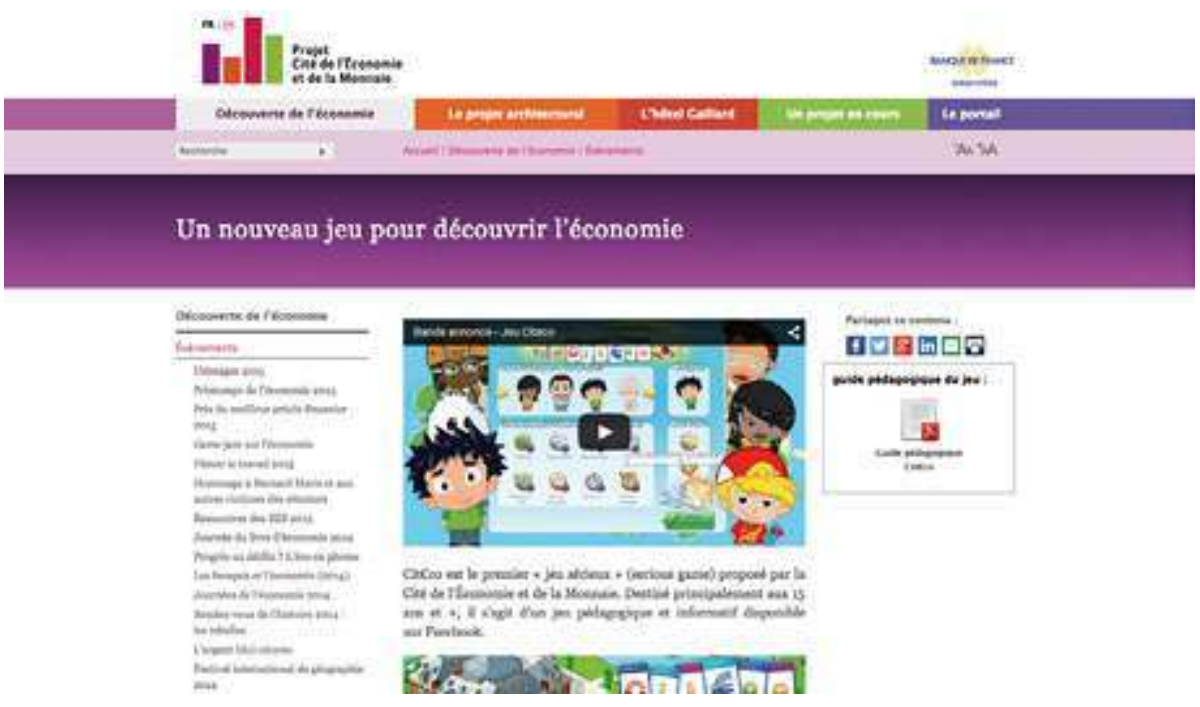

(c) Cité de l'économie et de la monnaie 


\section{Les principes de la médiation}

19 Une attention particulière sera portée à la forme des dispositifs muséographiques et des médiations. Les nombreux dispositifs numériques répartis dans l'exposition permanente auront pour objectif de mobiliser le visiteur, en lui proposant d'éprouver de multiples situations, notamment sous la forme de jeux de rôles immersifs. Tout en incarnant des concepts économiques immatériels, ces dispositifs mettront en scène des situations connues, parfois issues du quotidien des visiteurs, pouvant placer le joueur dans une posture réflexive. Cette muséographie "impliquante" devrait successivement mettre le visiteur en état de questionnement, en situation de découverte, de réflexion personnelle. Cette succession de postures, mobilisant différents registres (cognitifs, affectifs, émotionnels...) vise à engendrer une meilleure compréhension du sujet exposé. Invité à choisir, agir, expérimenter, le visiteur pourra ainsi personnaliser son parcours, s'appropriant les contenus et construisant peu à peu son autonomie. Cet angle d'approche vise une participation active du public à la construction de sa propre mémoire de l'exposition.

Ces dispositifs muséographiques devraient également permettre d'accroittre l'interaction entre les individus, matérialisant ainsi un des concepts de base pour comprendre l'économie: celle d'une société d'acteurs en situation permanente de production, d'échanges et d'interdépendances.

Par ailleurs, s'adressant en priorité aux adolescents, la Cité prendra en compte leurs modes de fonctionnement et besoins : les conduire à expérimenter et à être impliqués afin de favoriser leur autonomie, leur proposer d'utiliser des outils multimédia au cours de la visite, les mobiliser sur un temps relativement long dans des projets pluridisciplinaires qui les valoriseront dans leur environnement personnel. Le développement de serious games ${ }^{4}$, dans plusieurs modules muséographiques du parcours permanent, visera également la création d'une dynamique de visite où s'équilibrent le ludique et le didactique. Certains de ces jeux seront d'ailleurs déployés sur Internet et permettront d'enrichir l'expérience avant, pendant, ou après la visite : Cit€co, le premier "jeu sérieux" proposé par la Cité de l'économie sur la gestion d'entreprise et le développement durable est d'ores et déjà disponible sur www.citedeleconomie.fr/Unnouveau-jeu-pour-decouvrir-l.

Malgré une forte présence des outils numériques et interactifs dans le parcours permanent (tables tactiles, projections vidéos, écrans, ambiances sonores...), la Cité de l'économie a choisi d'accorder une place importante à la médiation humaine. Des médiateurs seront répartis dans les salles d'exposition permanente, dans les ateliers pédagogiques et les espaces d'animation intégrés au parcours permanent, l'auditorium (environ 100 places) ou encore le centre de ressources dédié à la pédagogie de l'économie.

La même philosophie, intégrant la participation des individus, la co-construction du discours, le principe de réflexivité, devrait être développée dans les médiations humaines. Tout en s'adaptant à leurs publics, les médiateurs auront pour mission de les accompagner dans leur découverte de la Cité, en problématisant les parcours et en choisissant le vocabulaire et le niveau de complexité requis. Sur la base d'un dialogue faisant émerger les questionnements personnels, privilégiant l'échange et les débats, les médiateurs auront la mission d'élaborer les visites avec les publics, l'objectif étant de les rendre peu à peu autonomes dans leur pratique de la Cité. Offrir aux plus grand nombre 
la possibilité d'acquérir et de s'approprier des outils leur permettant d'améliorer leur compréhension du monde économique sera ainsi une ambition centrale du programme de médiations de la Cité de l'économie et de la monnaie.

"Comment reconstruire la confiance ?", un projet mené par des lycéens de l'académie de Lyon : Confiance et monnaie (Terminale ES, Lycée La Martinière Duchère à Lyon, professeur, Dominique Chazal).

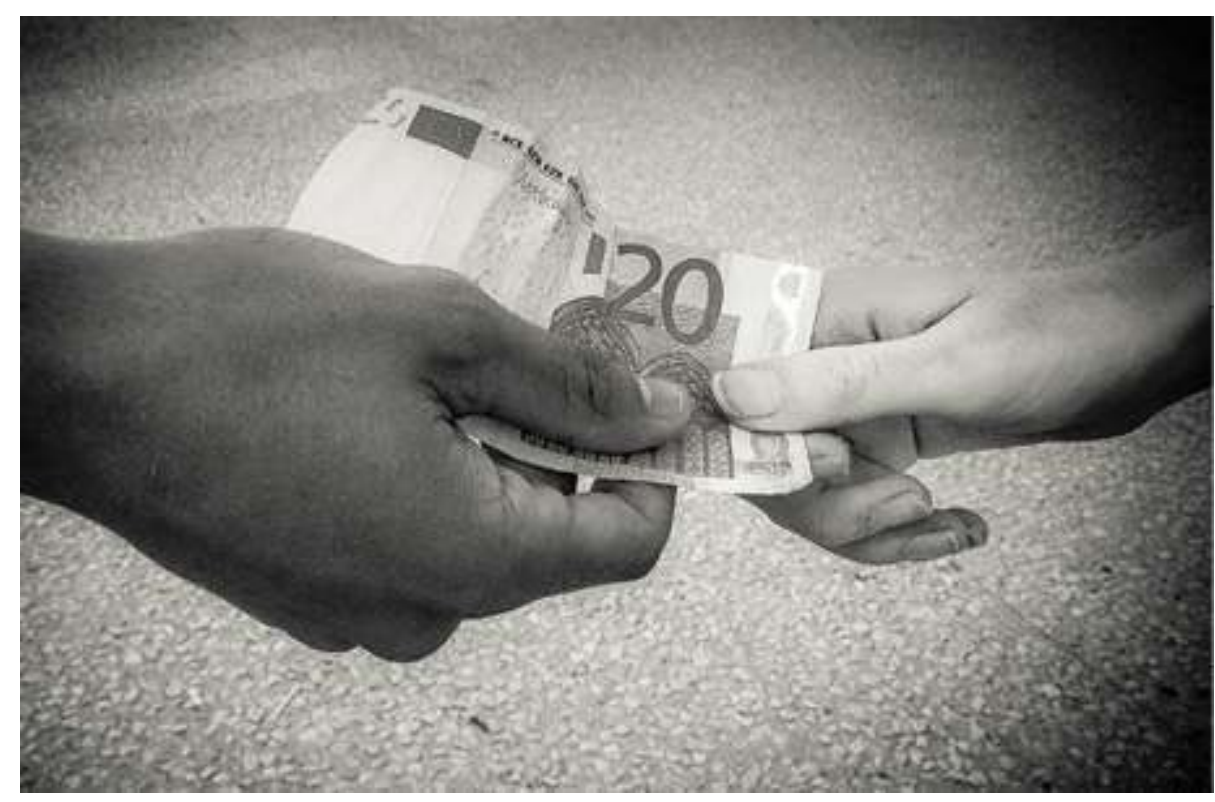

(c) DR

\section{Un exemple d'action de préfiguration}

En attendant l'ouverture, l'équipe projet de la future Cité de l'économie et de la monnaie développe et met en œuvre des actions de médiation hors-les-murs, dites de préfiguration. Ces dernières reflètent certaines des ambitions pédagogiques de la Cité de l'économie et permettent de tester et orienter les futurs dispositifs de médiation, à l'instar du site Internet, avec ses outils pédagogiques déjà en ligne ${ }^{5}$.

À l'occasion des Journées de l'Économie 2013, manifestation qui se tient chaque année à Lyon au mois d'octobre/novembre et qui regroupe, pendant 3 jours, de nombreux chercheurs, enseignants, étudiants et élèves, un projet pilote a été initié. Les Journées de l'Économie sont organisées depuis 2008 sous l'égide d'un comité scientifique par Pascal Le Merrer, enseignant à l'ENS Lyon, responsable des sites SES-ENS, touteconomie.org et journeeseconomie.org.

26 Partant du thème annuel des Journées de l'Économie en 2013, "Comment reconstruire la confiance ?", la Cité de l'économie a regroupé, dès la rentrée de septembre : - quatre classes de lycées de l'académie de Lyon: une Terminale ES, deux Terminales et une 1ère STMG (Sciences et Technologies du Management et de la Gestion);

28 - des équipes pédagogiques pluridisciplinaires regroupant des enseignants de SES, d'ÉcoGestion et de Philosophie, et des inspecteurs pédagogiques ;

29 - un photographe professionnel pour accompagner les classes lors d'ateliers de prises de vues photographiques ; 
30 réalisé une présentation du matériel et de quelques techniques de prises de vues photographiques'. Ces apports préalables et l'encadrement pédagogique des enseignants ont fourni les ingrédients de base aux élèves pour qu'ils se saisissent du sujet et arrivent à le problématiser. L'appropriation s'est opérée lors de la première séance de prises de vues, qui a permis l'émergence des premiers questionnements personnels. Confrontés à un terrain connu - les abords de leur établissement scolaire - les élèves l'ont découvert sous un angle nouveau, par le biais de l'appareil photographique et du sujet sur la confiance. La matérialisation du sujet s'est réalisée à cet instant, l'objectif de l'appareil aidant les élèves à choisir les éléments évoquant au mieux, pour eux, la confiance. Ce nouveau regard porté sur leur environnement immédiat a déclenché le débat, les faisant s'interroger sur eux-mêmes et sur l'importance de la confiance au quotidien.

L'exposition de photographies des lycéens lors des Journées de l'Économie.

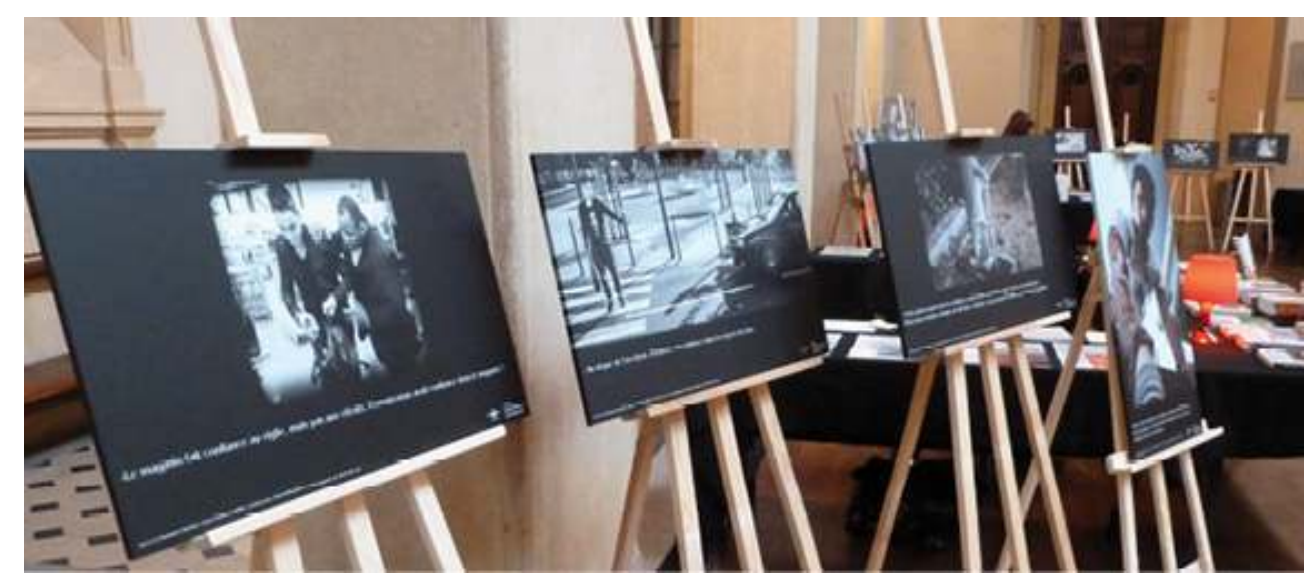

(c) Xavier Limagne

Entre ce premier temps de prises de vues et la seconde séance, les représentations préalables des élèves sur la confiance dans leur quotidien ont évolué. Lors de la seconde 
séance, les élèves ont réussi à faire la synthèse entre leur bagage personnel mis en mouvement et le thème de la confiance. Leur créativité a pu s'exprimer et leurs clichés traduire l'importance de la confiance dans les relations amoureuses, l'espace urbain lorsque l'on traverse la rue sur un passage piéton par exemple - et les gestes du quotidien, notamment dans l'utilisation de la monnaie. Enfin, la dernière séance a été consacrée à la sélection des visuels, aux retouches et à la rédaction des légendes des photographies.

Les photographies ont également été présentées dans des commerces de la Duchère.

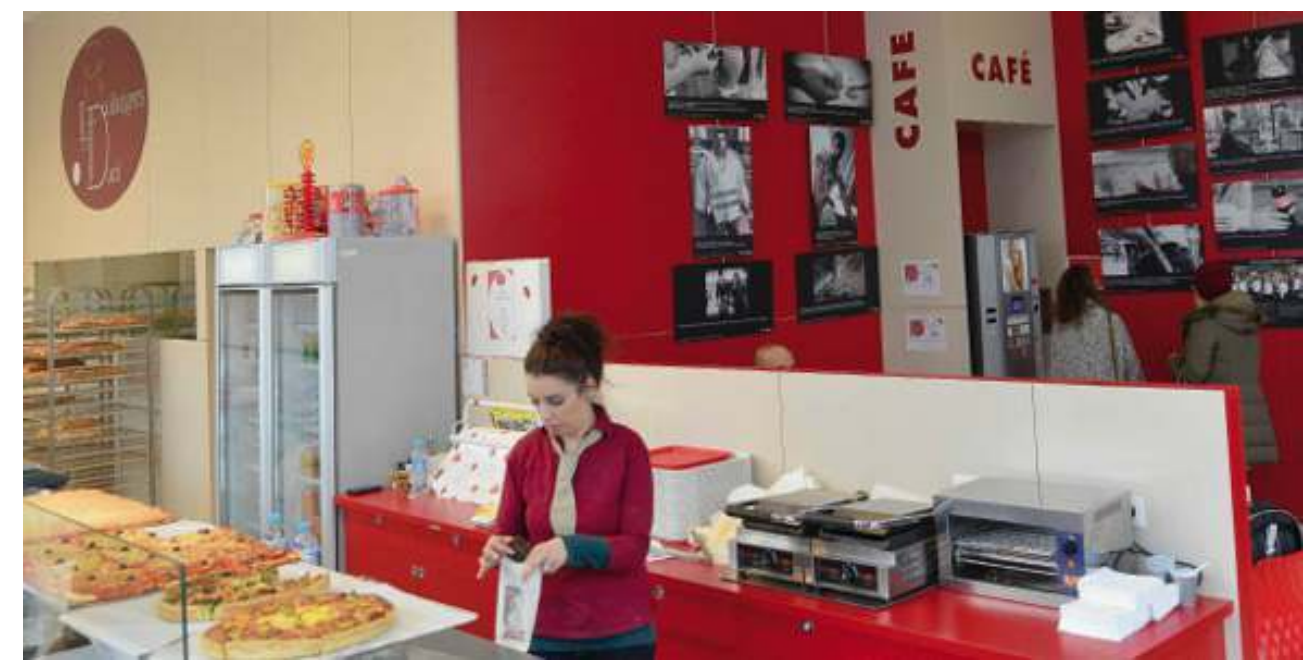

(c) Denis Lafontaine 
Cette dernière étape, inattendue et accueillie avec joie, incarne le processus d'autonomisation du projet et son intégration dans le quotidien réel de ses participants.

Au-delà du sentiment de fierté des élèves et de l'intérêt porté à cette démarche par les enseignants, les inspecteurs pédagogiques et les experts, quel bilan et enseignements peut-on tirer de ce projet de médiation en économie?

\section{L'heure du bilan} $\mathrm{du}$ dispositif. Devenant les acteurs principaux au sein d'un environnement et d'un cadre de référence que l'institution leur proposait de découvrir (ici le thème de la confiance), ils ont pu développer leur imagination et leur créativité. Cette liberté relative a instauré une relation de réciprocité entre, d'une part, l'institution qui leur a proposé de prendre la parole et, d'autre part, chacun des élèves qui a participé, accepté les règles du jeu, et produit un contenu qu'il a transmis en retour à l'institution, cette dernière s'employant à le valoriser. des acteurs et du projet a pu s'opérer. Ce projet a donc permis d'établir un dialogue entre les partenaires et d'incarner ainsi une forme de "parole circulaire"10.

Les opinions exprimées dans ce texte sont de la seule responsabilité de l'auteur et n'engagent pas l'Institution pour laquelle elle travaille. 


\section{NOTES}

1. Sur la culture économique des Français, voir notamment : Les Français et l'économie Synthèse de l'enquête quantitative Banque de France/TNS Sofres. Journées de l'Économie à Lyon, Bulletin de la Banque de France, $n^{\circ} 90$, 4e trimestre 2012 ; Les Français et l'économie L'enseignement de l'économie devrait tenir une place plus importante dans le système éducatif, Bulletin de la Banque de France, $\mathrm{n}^{\circ} 194$, 4e trimestre 2013. Concernant le rapport Guesnerie : www.idies.org/public/fichiers\%20joints/Rapport_d'audit_sans_annexe.pdf

2. www.journeeseconomie.org www.leseconomiques.fr www.festivaldeleconomie.org www.centregeopolitique.com www.fig.saint-die-des-vosges.fr www.rdv-histoire.com.

3. Muséo Interactivo de Economia à Mexico, www.mide.org.mx/mide/

4. Fourquet-Courbet, M.-P. et Courbet, D. Les serious games, dispositifs numériques de médiation : processus sociocognitifs et affectifs dans les usages et les effets sur les publics, Culture et Musées, n²2, 2014, pp. 165-190

5. Voir notamment la rubrique Découverte de l'économie : www.citedeleconomie.fr/-Une-citede-l-economie-

6. http://dessinemoileco.com/

7. Pour consulter l'album en ligne : www.citedeleconomie.fr/Photographie-moi-la-confiance

8. http://dessinemoileco.com/pas-deconomie-sans-confiance/

9. Denis Lafontaine : www.dlafontainephotographe.com/

10. Chaumier, S. De la conscience des sciences à l'enrichissement des âmes : du musée de science au musée de sciences et de sociétés, in Côté, M. (dir.) La fabrique du musée de sciences et de sociétés. Paris : La Documentation française, 2011, pp. 15-24.

\section{RÉSUMÉS}

Après avoir présenté le projet de création à Paris d'un espace muséal spécifiquement dédié à l'économie et organisé autour de formes de médiation qui font notamment appel à des jeux de rôle immersifs et à des procédés muséographiques conduisant le visiteur à la construction de sa propre mémoire de l'exposition, l'auteur relate une action de préfiguration qui a permis de tester ces dispositifs de médiation auprès du public lycéen.

\section{INDEX}

Mots-clés : cité de l'économie et de la monnaie, Paris 


\section{AUTEUR}

\section{CHARLOTTE DRAHÉ}

responsable des publics, service des contenus pédagogiques et muséographiques, direction du projet de la Cité de l'économie et de la monnaie, Banque de France

charlotte.drahe@banque-france.fr 